\title{
Stochastic Analysis of the Effect of Using Harmonic Generators in Power Systems
}

\author{
Mohsen Abbas Pour Seyyedi and Amir Hossein Jahanikia \\ Mefragh Company \\ Iran
}

\section{Introduction}

Switch mode electronic devices including Compact Fluorescent Lamp (CFL) and personal computers introduce capacitive power factor and current harmonics to the power system. Since middle 80's and with the expanding use of nonlinear switch mode electronic loads, concerns arose about their effect on the power systems. In many IEEE documents, it is recommended to study the effect of electronic loads. Switch mode devices have a capacitive power factor between 55 and 93 percent (Allexperts), which can cause the increase of reactive power and power loss. The power loss in an office building wirings due to the current harmonics may be more than twice that of the linear load equipment (Key et al., 1996). Capacity of the transformers may be reduced more than 50 per cent in the presence of harmonic components (Schneider, 2009).

CFL is a more efficient and durable replacement of the traditional incandescent lamp. Replacing traditional light bulbs by CFLs has several advantages including energy saving, increase in the capacity of plants and distribution transformers, peak shaving, less carbon emission and customer costs. On average, 20 percent of the total use of electricity is consumed in lighting (Michalik et al., 1997), (Tavanir). However, the increase in the number of electronic devices especially the CFLs in power systems must be carefully planned. Replacing the incandescent light bulbs with CFLs means replacing the system's major Ohmic load with a capacitive load of high frequency harmonic components. In areas where lighting is a major use of electricity, e.g. places where natural gas or other fossil fuels are used for heating purposes, unplanned replacing of incandescent lamps with CFLs can introduce unexpected negative effects on the system. Also, in areas with a considerable number of other switch mode devices e.g. commercial areas with many office buildings it is important to plan the number of CFLs carefully. Most of the present studies on the effect of switch mode devices are based on tentative experiments and power factor measuring before and after using the devices in the power system (Gonos et al., 1999), and proposing a model for the network has been less discovered.

In order for studying such effects, it is better to classify the system equipment to the substation equipment and consumer side equipment. Dramatic changes in power quality indicators of the distribution systems may cause disorders or even damages in the consumer equipments. Such disorders are especially important for sensitive appliances such as medical and hospital devices. 
In this chapter we review our novel approach for studying the effect of switch mode devices and present a novel stochastic modelling approach for analysing the behaviour of the power system in the presence of switch mode devices. We also study the major KPI of the power system and study how these KPI will be affected by adding the current harmonics. Section 2 presents how we obtain an accurate model for CFL based on circuit simulation. This section also defines a general circuit model for the harmonic generating devices. Section 3 presents our novel approach for stochastic modelling of the power system behaviour. In section 4 we summarize the major power system KPI on both substation and consumer sides. We also discuss how the switch mode devices may affect the devices on each side. Section 5 presents our approach for simulating the power system behaviour. Conclusion and discussion are presented in section 6 .

\section{Modelling of switch mode devices}

This section studies the general specifications of switch mode devices. We simulate a CFL ballast circuit in SPICE software. We also present the device model for a personal computer. Based on these models, we develop a general circuit model to simulate the behaviour of all switch mode capacitive devices. Without circuit simulation, it is not possible to provide an accurate model representation in the power system. In contrast with the models that are based on measuring and estimating the device characteristic, this approach gives much more accurate results. The accuracy of this approach can be chosen at the desired level.

\subsection{Simulation of CFL ballast circuit in SPICE}

The common $220 \mathrm{~V}$ power system voltage is not enough to start the fluorescent lamps. Therefore, CFLs include a ballast circuit for providing the starting high voltage. In traditional fluorescent lamps, inductive ballasts are widely utilized. However, electronic ballasts which are used in CFLs have much better quality (Aiello et al., 2008). Electronic ballasts are composed of a rectifier and a DC-AC converter. Fig. 1 shows the general block diagram of a ballast circuit.

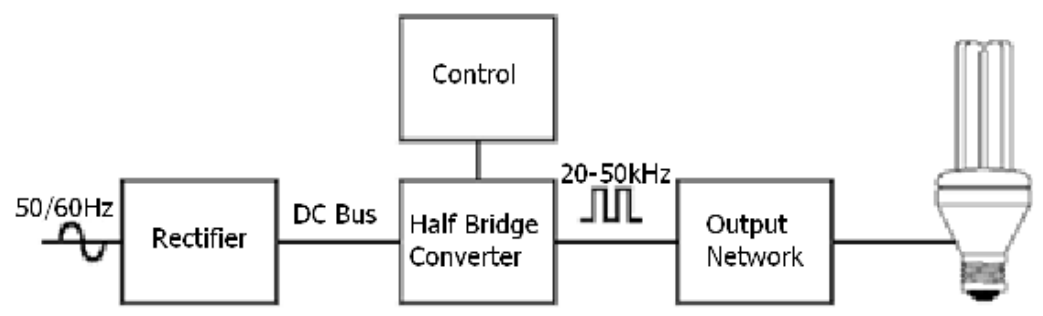

Fig. 1. Block diagram of a CFL ballast circuit. Figure courtesy of (Sasaki, 1994).

Several circuits are simulated in SPICE software for this project. Fig. 2 shows one sample CFL ballast circuit model in SPICE. This circuit is similar to that of (Sasaki, 1994) with slight changes. The input full wave rectifier and the large input capacitor make the current have narrow high peaks at short intervals and almost zero value elsewhere. Fig. 3 shows the output voltage and current of the circuit in Fig. 2.

Frequency analysis shows that the CFL current is made up of odd harmonic components of the main frequency ( 50 or $60 \mathrm{~Hz}$ ). The CFL is modelled by a number of current sources with 
the proper harmonic values. Equation 1 shows the mathematical model for a CFL when the voltage is assumed to be a cosine function.

$$
\begin{aligned}
& v_{C F L}=V \cos (2 \pi f t) \\
& i_{C F L}=\sum_{n=0}^{\infty} I_{2 n+1} \cos \left[2 \pi(2 n+1) f t+\Phi_{2 n+1}\right] \approx \sum_{n=0}^{4} I_{2 n+1} \cos \left[2 \pi(2 n+1) f t+\Phi_{2 n+1}\right]
\end{aligned}
$$

The more the number of harmonics is, the more accurate the model will be. In this study we use the first five odd harmonics $(1,3,5,7$, and 9). A schematic of the model is shown in Fig. 4. The power factor of this circuit is $93 \%$. In order for having a flexible model for different market suppliers, the power factor is chosen flexible in the simulation experiments.

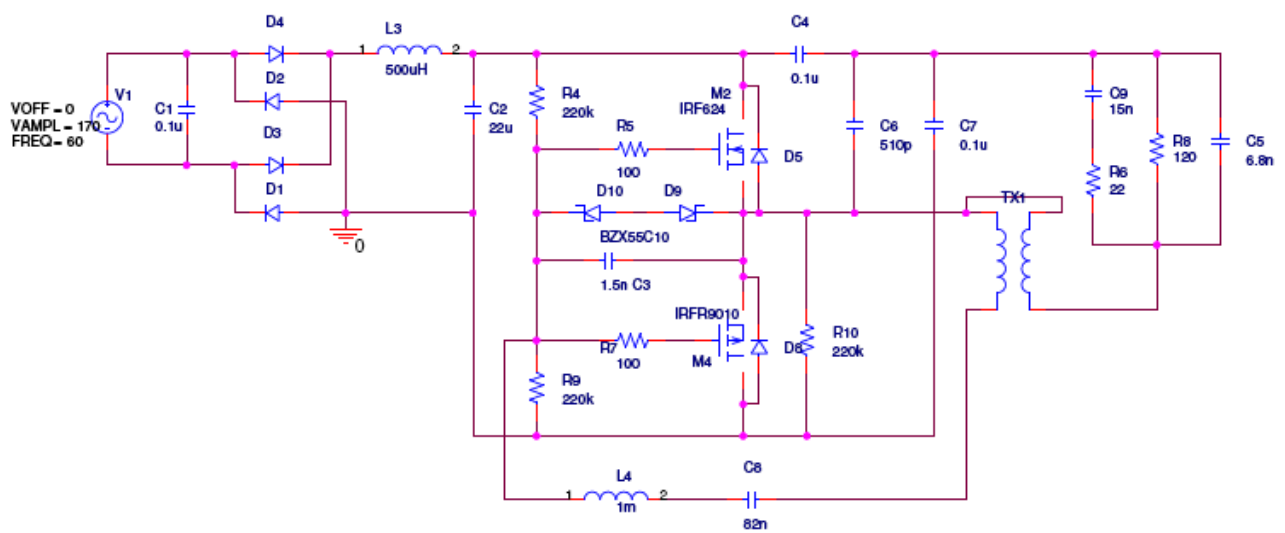

Fig. 2. Simulation of a sample ballast circuit in SPICE.

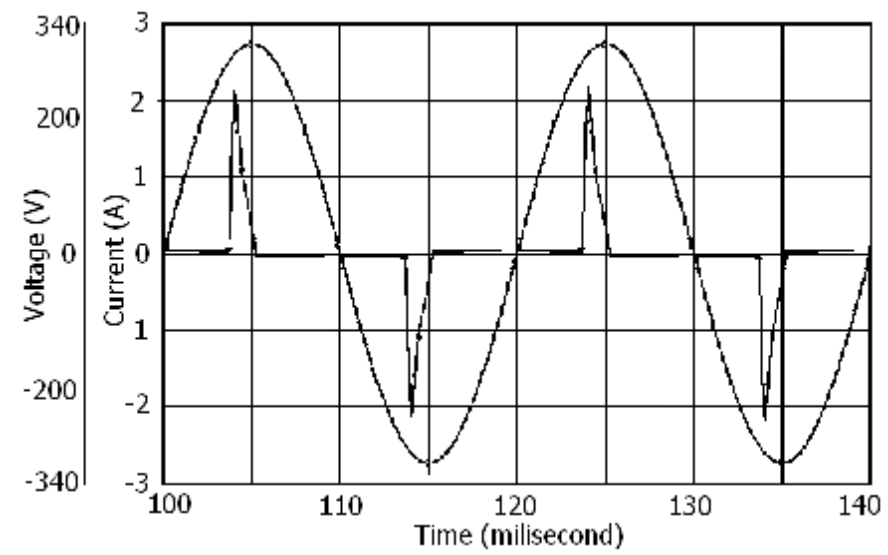

Fig. 3. Sinusoidal voltage and resulting current waveshape for a sample CFL ballast circuit. 


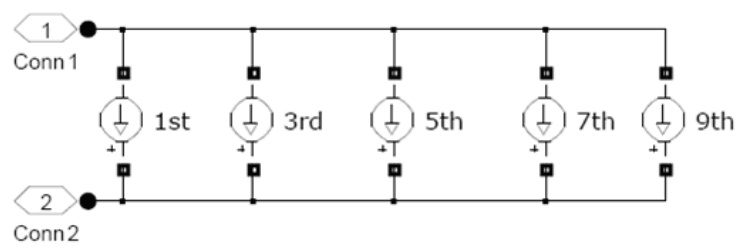

Fig. 4. Circuit model of a switch mode device.

The values of the current and phase in equation 1 are summarized in Table 1 for the circuit in Fig. 2.

\begin{tabular}{|l|l|l|l|l|l|}
\hline Current Harmonic & First & Third & Fifth & Seventh & Ninth \\
\hline Peak $\mathbf{I}_{\mathbf{2 n + 1}}(\mathbf{A})$ & 0.2 & 0.182 & 0.162 & 0.138 & 0.112 \\
\hline Phase $\boldsymbol{\Phi}_{\mathbf{2 n + 1}}(\mathbf{R a d})$ & 0.260 & 3.499 & 0.609 & 4.000 & 0.799 \\
\hline
\end{tabular}

Table 1. Peak value and phase of the current harmonics for the sample CFL of Fig. 2.

We name the overall current phase lag as central phase lag $\Phi_{c}$.

\subsection{Circuit model for other electronic devices}

Personal computers and other electronic equipment such as printers, etc. generate current harmonics in the power system too, because they all include a rectifier. The harmonic components of personal computers are calculated and provided in the literature (Key et al., 1996). Fig. 5 shows the relative value of these components. Therefore, we can use a similar model to that of Fig. 4 for modelling such electronic devices.

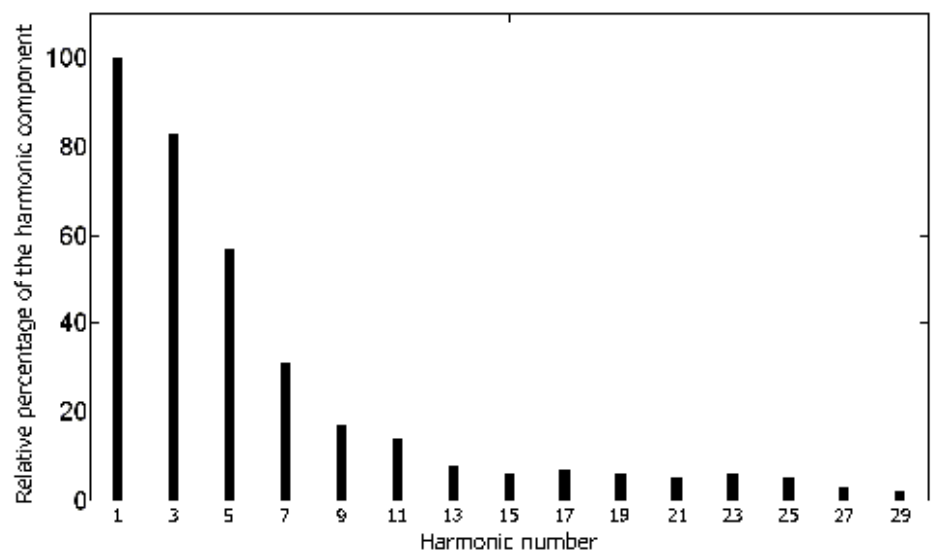

Fig. 5. Relative values of the current harmonics for a personal computer.

\section{Stochastic modelling of switch mode devices in power system}

Phase of a harmonic generating device is not a constant value. But it is a random variable that varies in a specific range that can be provided by the manufacturer. Therefore, the model in equation 1 will be modified to that of equation 2 . 


$$
i_{m}=\sum_{n=0}^{4} I_{2 n+1} \cos \left[2 \pi(2 n+1) f t+\Phi_{2 n+1}+(2 n+1) \times \Delta \Phi_{m}\right]
$$

In this model, $m$ is the device number in the network. In practice, the total number of devices $M$ is a large number. For each device we have a phase shift $\Delta \Phi_{m}$ from the central phase. Distribution of $\Delta \Phi_{m}$ can be assumed to be uniform or Gaussian depending on the manufacturer's datasheet. In other words for the uniform distribution:

$$
\Phi_{c}-\Phi_{\max }<\Delta \Phi_{m}<\Phi_{c}+\Phi_{\max ,} \quad P\left(\Delta \Phi_{m}=\phi\right)=\frac{1}{2 \Phi_{\max }}
$$

In the above equation, $\Phi_{\max }$ is the maximum phase shift from the theoretical phase lag $\Phi_{c}$. If the probability distribution is Gaussian, $\Delta \Phi_{m}$ is obtained from equation 4 :

$$
P\left(\Delta \Phi_{m}=\phi\right)=\frac{1}{\sqrt{2 \pi \sigma^{2}}} e^{(\phi-\bar{\phi})^{2} / 2 \sigma^{2}}
$$

Where $\varphi$ and $\sigma$ are the mean and variance of the phase lag. The current value for a large number $M$ of CFLs with the above specifications is equal to $i$ in equation 3:

$$
i=\sum_{m=0}^{M} i_{m}=\sum_{m=0}^{M} \sum_{n=0}^{4} I_{2 n+1} \cos \left[2 \pi(2 n+1) f t+\Phi_{2 n+1}+(2 n+1) \times \Delta \Phi_{m}\right]
$$

Finding the probability density function, expectation and variance of current in the above equation is complicated (if possible!). Instead, we rely on numerical simulation to find the $p d f$ of power system current. In a sample experiment, our power system is composed of a thousand CFLs. The average phase lag of these CFLs is fifteen degrees and has a uniform distribution of range $15 \pm 10$ degrees. We use a Parzen window (Duda et al., 2007) approach to find the $p d f$ of the peak output current. Fig. 6 shows the result. The mean value and standard deviation of the current in this experiment are 611.5 and 2.45. We may notice that if the variance in the phase shift is not considered, meaning that we do not assume the CFL phase to be a random variable, the peak current will be equal to 701 Amperes. When we dimension the network, this means at least $13 \%$ more capacity. Different results can be obtained for different values of phase range for the electronic devices.

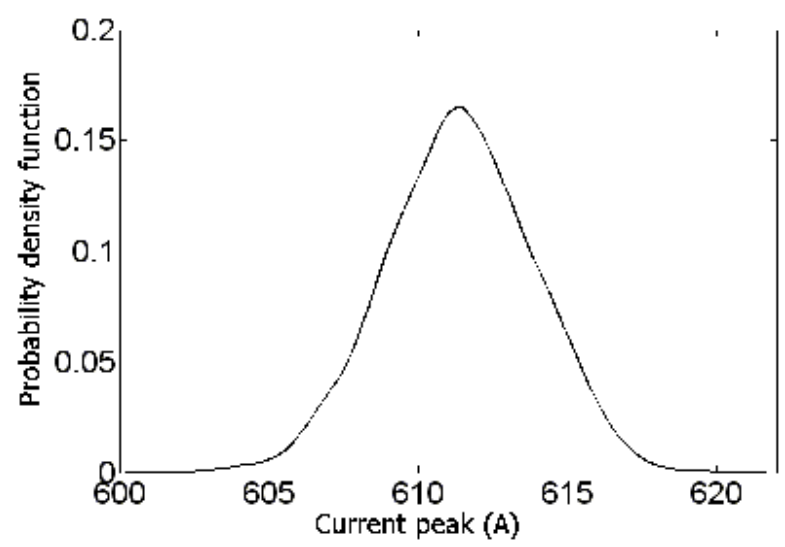

Fig. 6. Relative values of the current harmonics for a personal computer. 


\section{Effect of switch mode devices in power system}

The main devices in any power systems include thermal loads, electronic devices and inductive loads. Thermal loads, including the traditional lighting, can be modelled as a simple resistance. In regions where a considerable part of heating is based on electricity, heating constitutes a large share of the thermal load. Electronic devices include television and personal computer. The most common inductive appliances are cooling devices, which are air conditioning systems and refrigerators. Different values of power from $150 \mathrm{~W}$ to 2000W are used for induction motors, depending on the application type and geographical region of use.

In this section we take a closer look to the different equipment and the effects of the harmonics on individual devices on both distribution and consumer sides. This section mainly focuses on theoretical study of the effects of the harmonics on the key performance indicators rather than practical measurements. Fig. 2 shows the fundamentals of the analysis in the rest of this section.

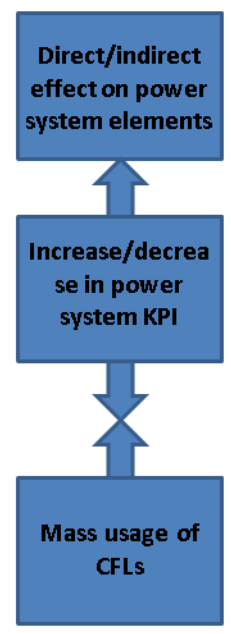

Fig. 7. Relation between using CFL (as a switch mode device) and the power system equipment.

\subsection{General power system KPI}

The most important and most useful KPI of the power system include:

\subsubsection{Total Harmonic Distortion (THD)}

THD is defined as in equation 6:

$$
T H D=\frac{\sqrt{\sum_{n=2}^{\infty} V_{n}^{2}}}{V_{1}}
$$


This factor may be calculated for voltage, current or power. Current THD is normally much higher than the voltage THD for electronic devices and is more than $100 \%$.

\subsubsection{Crest Factor}

Crest Factor is the indicator that shows the ratio of the peak value of the wave shape to its average value in a time interval (rms) ${ }^{1}$, as shown in equation 7 .

$$
C=\frac{|x|_{\text {peak }}}{x_{r m s}}
$$

This factor is especially important for the analysis of the effect on protective and control equipment such as relays.

\subsubsection{Power Factor}

Power Factor is caused by the input rectifying capacitor of the ballast circuit. The capacitive characteristic of CFL is an advantage in systems with a high inductive load.

\subsection{Power substation equipment}

This section studies the effect electronic devices on the substation equipments, which provide the low voltage power to the end users and can be classified to the following categories:

\subsubsection{Transformers}

Transformers are used in the distribution system in order to change the levels of voltage and current in the low voltage scales. These may also include the power and instrumentation transformers and Auto-Boosters. In transformers, both the core and the wires are sensitive to the change of the power KPI. The harmonics have the following different effects on transformers.

Transformer loss, which is obtained as in equation 8 :

$$
P_{T}=P_{N}+P_{L L}
$$

In equation $8, P_{N}$ is the "no-load" loss and $P_{L L}$ is the "full-load" loss. The no-load loss depends on the voltage and core material. The full load loss is defined as in equation 9:

$$
P_{L L}=P_{D C}+P_{E C}+P_{O S L}
$$

In equation $9, P_{D C}$ is the DC resistance loss, $P_{E C}$ is the eddy current loss and $P_{O S L}$ is the stray loss. Eddy current, which is proportional to the square of frequency, is caused by skin effect and proximity effect. Therefore, the current harmonics increase the eddy current loss dramatically. This increase results in the increase in temperature and hence reducing the transformer lifetime (Ashok).

Lifetime of a transformer depends on the functioning situations such as loading percentage and functioning temperature. Current harmonic components can increase the RMS value of

\footnotetext{
${ }^{1}$ Root Mean Square
} 
the current and consequently the resistive power loss. The heat also remains in the surrounding air and affects the transformer lifetime. In the delta-wye connections in the transformers, the harmonic components of current start rotating in the wye side and cause heat generation and reduce transformer capacity. This occurs for the 3rd, 9th and 15th harmonic components. The current harmonics also cause saturation of the transformer.

In order to control the effect of current harmonics on transformers and electromotors, it is recommended not to have a current harmonic component more than $5 \%$ of the transformer's nominal current in the ANSI/IEEE C57.12.00-2000 and IEC60076 standards (Sadati et al., 2008). The K-Factor, which is defined in equation 10, identifies the relation between transformer design and increase in the electronic devices.

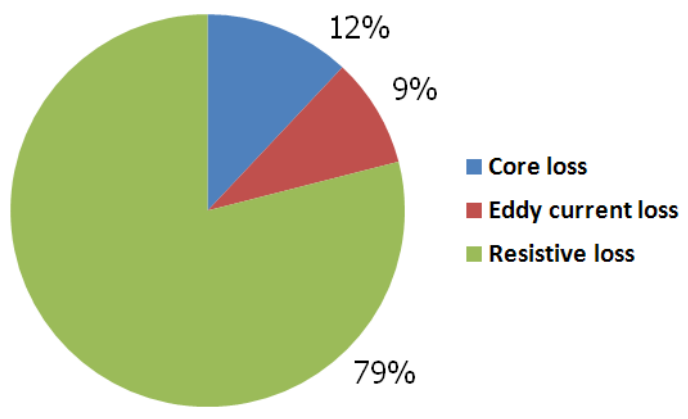

Fig. 8. Comparing different losses in the transformer core.

$$
K-\text { Factor }=\sum_{h=1}^{\infty} I_{h}(p h)^{2} h^{2}
$$

In equation 10, $h$ is the harmonic order and $I_{h}$ is its current component in per unit. The definition of K-Factor may be different in different standards. For example, in BS7821 standard it is defined as in equation 11.

$$
K-\text { Factor }=\left[1+\frac{e}{1+e}\left(\frac{I_{1}}{I}\right)^{2} \sum_{n=2}\left(n^{q}\left(\frac{I_{n}}{I_{1}}\right)^{2}\right)\right]^{0.5}
$$

\subsubsection{Transmission systems}

Transmission systems include power cables and wires. Transmitters are the conductors which are used in power system. Because of the skin effect (Hightech), the resistance of conductors increases with the increase of frequency. Because of the small high frequency components of the electronic devices, using these devices has a small effect on the conductors. Simulations show that the current amplitude for a sample CFL in frequencies between 1000 and 1500 Hertz (harmonics 20th to 30 th) is less than $1 \%$ of the nominal value. Therefore, capacity reduction of cables and wires will be between $1 \%$ and $6 \%$ of conductor capacity in the worst case (100\% replacement) (Vapopoulos, 1964). 


\subsubsection{Electronic devices:}

Electronic devices including the automation or control devices and the equipments that are used for stabilizing the power systems such as PLC transmitters and RTU equipment. As the electronic devices are used for transmitting and receiving data, high frequency current can cause disorders in their functioning, such as increased noise level in communication systems. Such devices are usually installed close to power system equipment such as transformers.

\subsubsection{Control and protection systems}

Control and protection system, such as fuses, relays and circuit breakers, which control or guard the power systems. Current harmonics in the system may cause pre-heating in the fuse and problems in its function. Fuses may also be affected by the skin effect and the resulting heat may cause their malfunctioning. In circuit breakers, which work based on $d i / d t$, current harmonics may cause unexpected faults. Here, the peak factor is important as well. The solenoids may also be damaged because of the harmonics. Delay in solenoid's functioning may cause sparks and damage. Vacuum circuit breakers are less sensitive to the harmonics. For relays, changes in the zero point may case improper functioning. These effects must be identified via practical experiments. Some manufacturers have presented "harmonic adaptive" models (Ashok).

\subsection{Equipment on the consumer side}

The consumer side equipments are classified into three different categories (Gowan, 2006):

\subsubsection{Electric machines}

Electric machines which are inductive devices and as confirmed by simulation using them together with electronic devices can improve the power quality indicators. The $n_{\text {th }}$ current component of an electric machine is obtained as in equation 12 :

$$
I_{n}=\frac{V_{n}}{n \cdot f \cdot L_{n}}
$$

In equation 12, $V_{n}$ is the $n_{\text {th }}$ voltage harmonic and $L_{n}$ is the inductance in $n_{\text {th }}$ harmonic (Markiewicz et al., 2004). $L_{n}$ increases because of the skin effect. Power loss of electric motors is obtained from equation 13:

$$
P_{h}=\sum P_{n}=k P_{r} \sum\left(\frac{V_{n}^{2}}{n^{1.5} \cdot V_{1}^{2}}\right)
$$

where $k=\frac{T_{s}}{T_{r}} \cdot \frac{E}{\left(1-S_{r}\right)\left(1-E_{r}\right)} \cdot T_{s}$ is the torque, $E_{r}$ is efficiency and $S_{r}$ is the slippage (Schneider 2010).

The heat generated by the harmonics may cause decreasing the motor lifetime. In addition, the eddy currents can generate heat in the motors, similar to the transformers. The heat generation is almost the same in synchronous and asynchronous motors. In the case when all incandescent lamps are replaced by CFLs, the performance of induction motors will be between 5 and 15 percent (Vapopoulos, 1964). For the asynchronous motors, IEC60892 standard is defined as in equation 14: 


$$
H V F=\sqrt{\sum_{h=2}^{13} \frac{U_{h}}{h^{2}}} \leq 0.02
$$

$U_{h}$ is the harmonic voltage of order $h$.

\subsubsection{Measurement devices}

Measurement devices: such as electricity meters, current transformers, voltage transformers and electronic instrumentation. The measurement transformer error is calculated as:

$$
K_{f}=C V K_{p}
$$

where $C$ is the ratio of real ratio to actual ratio (for current transformers), $V$ is the ratio of real ratio to actual ratio (for voltage transformers) and $K_{p}$ is angle correction coefficient (Hightech). The same as in power station transformers, high frequency current harmonics result in early saturation of transformer core and error in measurement. Electronic devices which work based on the zero crossing may also be affected by wave shape distortion. Errors may occur while calculating voltage and current rms values. In the inductive electricity meters (Temple, 1998) the error may be more. Changes in power factor and the THD affect the operation of these equipments.

\subsubsection{Communication devices}

Most communication systems are equipped with filters to reduce the noise generated by high frequency components. As the harmonics above $1500 \mathrm{~Hz}$ are negligible in electronic devices, they will practically not affect the communication systems. Similarly they will not affect electronic devices on the consumer side. Some malfunction of printers is reported (Abbaspour et al., 2009). Multimedia devices and televisions may be affected when used in the presence of harmonics. Distortion may be introduced by either the harmonics or the electronic ballast frequencies. All such devices are equipped with electromagnetic filters (EMC) which remove such effects. Personal Computers are sensitive to a voltage distortion of more than $5 \%$. However, the use of harmonic generating devices does not introduce more than $0.5 \%$ voltage THD.

As the CFL ballast circuits work in $40 \mathrm{KHz}$ frequency, they may affect hospital devices. Some distortions are reported in the functioning of CT devices (Abyaneh, 2004). Also because of the type of the CFL light, it is not recommended in operation rooms. The most important quality parameters which are considered here are the total power use, total harmonic distortion and transformer eddy current loss and hysteresis effect. Different measurements can explain the effect of harmonics. Total harmonic distortion (THD) is defined in equation 1 (Chapman).

\section{Simulating of power system}

In this section, we develop individual units of consumers developed, which are a certain combination of electric device models. These units are called with terms "home" and "office". These models are obtained using statistical data about different residential regions in Iran. In the simplest case, a home is a combination of four lighting loads and one induction motor. Fig. 9 shows this consumer unit simulated in Simulink (Mathworks). 


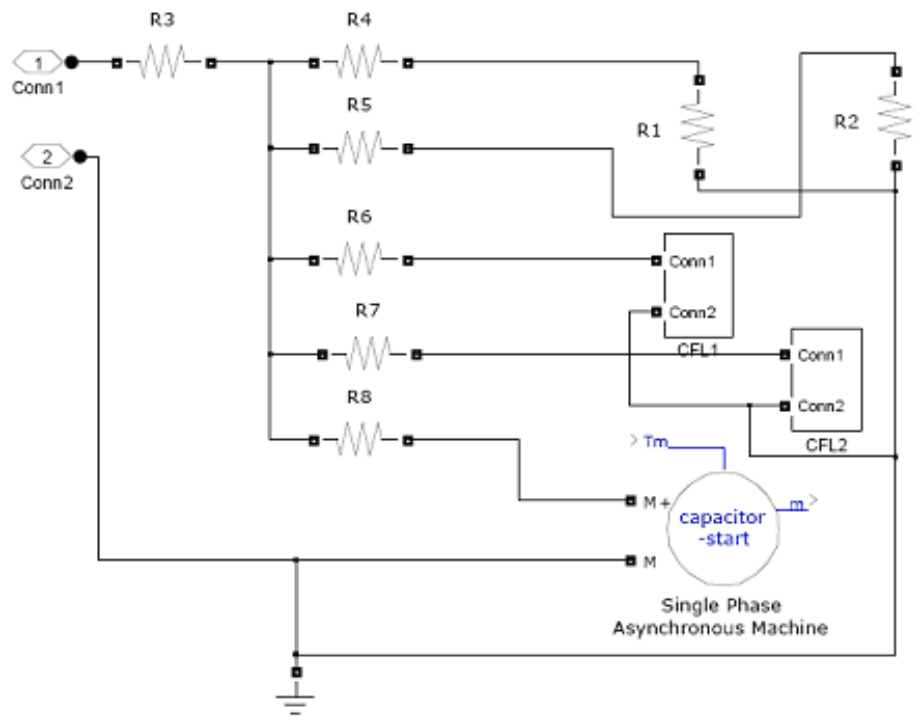

Fig. 9. "Home" unit with two CFLs, two lamps and induction motor.

Fig. 10 shows a sample commercial (office) consumer unit.

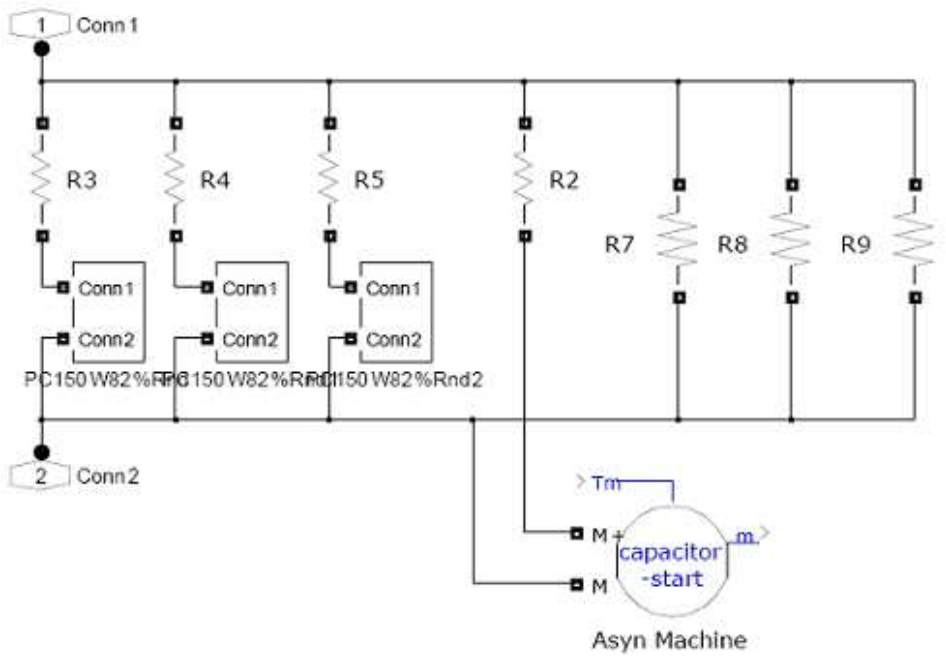

Fig. 10. "Office room" unit with four incandescent lamps, three PCs and a single phase asynchronous motor. 
Eddy current loss of transformer core depends on the squares of both current and frequency (Bird). Therefore, the core loss for different experiments is compared to each other using equation 16. $f_{h 1}$ and $f_{h 2}$ correspond to the frequency components of the different cases in comparison.

$$
\text { Relative core loss }=\frac{\sum_{h=1}^{N} f_{h 2}^{2} I_{h 2}^{2}}{\sum_{h=1}^{N} f_{h 1}^{2} I_{h 1}^{2}}
$$

A similar approach is used for the hysteresis effect. However, hysteresis effect comparison is obtained using equation 17 (Bird).

$$
\text { Relative hysteresis }=\frac{\sum_{h=1}^{N} f_{h 2} I_{h 2}^{1.6}}{\sum_{h=1}^{N} f_{h 1} I_{h 1}^{1.6}}
$$

\section{Conclusion}

A new approach is presented for the analysis of switch mode devices and their effect on distribution network. In this approach, model of electronic devices are derived from detailed circuit simulation and the results are transformed to a circuit model which is a combination of passive current circuits. The model contains the odd harmonics of the power system frequency. Based on this model, we developed a probabilistic model for the electronic device and used numerical calculation to estimate the probability distribution of the power system current as a whole. This model shows that adding electronic devices to the power system must be carefully planned so that the power system KPI do not exceed the recommended levels. This stochastic simulation is a strong tool to evaluate the situation of the power system and its capacity for growth. In case of mass use of electronic devices, additional capacity must be provided both for transformers and distribution lines, in order to avoid harms in the network via harmonic mitigation.

We also presented a list of the power system KPI and the devices that can be affected by the additional current harmonics. We classified the equipments into the feeding and consuming sides and studied the effects on each side separately. Mass usage of switch mode devices in different power systems must be planned carefully in order to avoid any unexpected negative effects on the other equipments in the system. The most vulnerable equipments of the power system are the ones which contain solenoids, such as transformers and measurement equipments, especially on the feeding side. Therefore, extra care and calculations are required in different power systems for a safe use of electronic devices. Electronic devices are affected by the harmonic distortion and may need to be either equipped with protective filters or be replaced by more advanced ones. Based on the power system KPI and the specification of the distribution and consumption devices we can use the stochastic analysis approach for dimensioning an optimal power system with respect to equipment costs and safety margins.

\section{Acknowledgment}

The authors of this paper wish to acknowledge the support of Mefragh Company, Tehran Iran, especially Mr. Hashem Khoee, president of the company. We also wish to thank Iran 
Energy efficiency Organization (IEEO - SABA) and especially Mr. Sajjadi for their continual support.

\section{References}

Key, T. S.; Jih-Sheng, L., (1996). Costs and benefits of harmonic current reduction for switch mode power supplies in a commercial office building, IEEE Tran. on Industry Applications, vol. 32, Issue 5, Sep/Oct 1996, pp. 1017-1025.

Schneider Electric. Electrical Installation Guide, 2009.

Michalik, G.; Khan, M. E.; Bonwick, W. J.; Mielczarski, W. (1997). Structural modeling of energy demand in the residential sectors. Elsevier, vol. 38, Number 6, November 1997, pp. 453-453.

http:/ /amar.tavanir.org.ir/

Gonos, I. F.; Kostic, M. B. ; Topalis, F. V. (1999). Harmonic distortion in electric power systems introduced by compactfluorescent lamps. International Conference on Electric Power Engineering, Budapest, 1999, pp. 295-300

Aiello, N.; Messina, S. (2008). VIPower: Self-Oscillating Converter Using VK05CFL for Compact Fluorescent Lamps.

Sasaki, R. I. (1994). The Impact of Electronic Ballast Compact Fluorescent Lighting on Power Distribution Systems. Purdue University School of Electrical Engineering, 1994.

Duda, R. O.; Hart, P. E.; Stork, D. G. (2007) Pattern classification. Wiley, ISBN 978-0-47105669-0

Sadati, S. B. ; Tahani, A. ; Darvishi, B. ; Dargahi, M. ; Yousefi, H. (2008). Comparison of Distribution Transformer Losses and Capacity under Linear and Harmonic Loads. in 2008 2nd IEEE International Conference on Power and Energy (PECon 08), Johor Baharu, Malaysia.

Ashok, S. Effect of power system harmonics on power system equipment. Nalanda Library Lectures.

Hightech Aus Co. Power factor correction and harmonics.

Vapopoulos, N. Th. (1964). Studies on harmonic analysis," Proc. Camb. Phil. Soc., 1964, pp. No 60, vol 465.

Gowan, C., Power quality and harmonics," Cornell University, 2006.

Markiewicz, H. Klajn; A. (2004). Power quality application guide. Wroclaw University of Technology, July 2004.

Schneider-Electric Publication (2010). The Electrical Installation Guide (EIG), ISBN: 978.2.9531643.3.6, version 2010.

Temple, D. (1998). Facilities Instructions, standards \& techniques. United States Department of the Interior Bureau of Reclamation, Power O\&M Bulletin No. 13, vol. 2-2, 1998.

Abbaspour, M. ; Jahanikia, A. H. (2009). Power Quality Consideration in the Widespread Use of Compact Fluorescent Lamps. in Proc. 2009 10th Int. Conf. Electrical Power Quality and Utilisation, Lodz, Poland, pp. 1-6.

Abyaneh, H. A. (2004). Analysis of harmonic distortion in power distribution systems. presented at the 9th Int. Conf. Power Distribution Systems, Tehran, Iran, 2004 (in Persian). 
Chapman, D. Rating of Transformer supplying harmonic loads. Leonardo Energy. Mathworks. www.mathworks.com

Bird, J. O. Electrical circuit theory and technology. Newnes, May. 


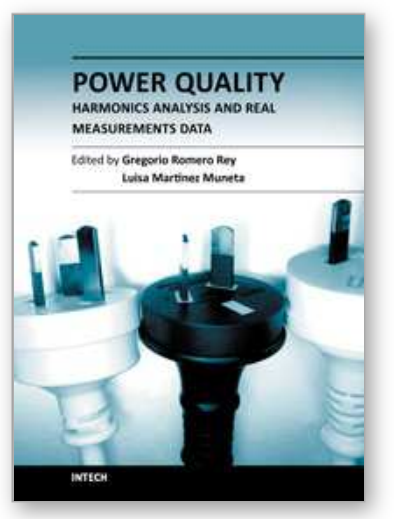

\author{
Power Quality Harmonics Analysis and Real Measurements Data \\ Edited by Prof. Gregorio Romero
}

ISBN 978-953-307-335-4

Hard cover, 278 pages

Publisher InTech

Published online 23, November, 2011

Published in print edition November, 2011

Nowadays, the increasing use of power electronics equipment origins important distortions. The perfect AC power systems are a pure sinusoidal wave, both voltage and current, but the ever-increasing existence of nonlinear loads modify the characteristics of voltage and current from the ideal sinusoidal wave. This deviation from the ideal wave is reflected by the harmonics and, although its effects vary depending on the type of load, it affects the efficiency of an electrical system and can cause considerable damage to the systems and infrastructures. Ensuring optimal power quality after a good design and devices means productivity, efficiency, competitiveness and profitability. Nevertheless, nobody can assure the optimal power quality when there is a good design if the correct testing and working process from the obtained data is not properly assured at every instant; this entails processing the real data correctly. In this book the reader will be introduced to the harmonics analysis from the real measurement data and to the study of different industrial environments and electronic devices.

\title{
How to reference
}

In order to correctly reference this scholarly work, feel free to copy and paste the following:

Mohsen Abbas Pour Seyyedi and Amir Hossein Jahanikia (2011). Stochastic Analysis of the Effect of Using Harmonic Generators in Power Systems, Power Quality Harmonics Analysis and Real Measurements Data, Prof. Gregorio Romero (Ed.), ISBN: 978-953-307-335-4, InTech, Available from:

http://www.intechopen.com/books/power-quality-harmonics-analysis-and-real-measurements-data/stochasticanalysis-of-the-effect-of-using-harmonic-generators-in-power-systems

\section{INTECH}

open science | open minds

\section{InTech Europe}

University Campus STeP Ri

Slavka Krautzeka 83/A

51000 Rijeka, Croatia

Phone: +385 (51) 770447

Fax: +385 (51) 686166

www.intechopen.com

\section{InTech China}

Unit 405, Office Block, Hotel Equatorial Shanghai

No.65, Yan An Road (West), Shanghai, 200040, China 中国上海市延安西路65号上海国际贵都大饭店办公楼 405 单元

Phone: +86-21-62489820

Fax: $+86-21-62489821$ 
(C) 2011 The Author(s). Licensee IntechOpen. This is an open access article distributed under the terms of the Creative Commons Attribution 3.0 License, which permits unrestricted use, distribution, and reproduction in any medium, provided the original work is properly cited. 